\title{
On some Lambert-like series
}

\author{
P. Agarwal, S. Kanemitsu and T. Kuzumaki
}

Dedicated to the memory of Alan Baker

\begin{abstract}
In this note, we study radial limits of power and Laurent series which are related to the Lerch zeta-function or polylogarithm function. As has been pointed out in [CKK18], there have appeared many instances in which the imaginary part of the Lerch zeta-function was considered by eliminating the real part by considering the odd part only. Mordell studied the properties of the power series resembling Lambert series, and in particular considered whether the limit function is a rational function or not. Our main result is the elucidation of the threshold case of $b_{n}=\frac{1}{n}$ studied by Mordell [Mor63], revealing that his result is the odd part of Theorem 1.1 in view of the identities (1.9), (1.5).

We also refer to Lambert series considered by Titchmarsh [Tit38] in connection with Estermann's zeta-functions.
\end{abstract}

Keywords. Lambert-like series; Lambert series; Estermann zeta function; trigonometric series; Dirichlet-Abel theorem; Riemann's posthumous fragment II.

2010 Mathematics Subject Classification. 11F03; 01A55; 40A30; 42A16

\section{Introduction}

As has been noticed in [CKK18], there are many instances in which the odd part, the first periodic Bernoulli polynomial, of the polylogarithm function of order 1 appears as a result of eliminating the real part, the log sine function. As has been developed, this kind of boundary behavior is essential in scientific as well as mathematical research [LiKa19], [LLK19], [WMK20]. As a typical example of a boundary function we consider the power series expansion of the monologarithm function

$$
-\log (1-z)=\sum_{n=1}^{\infty} \frac{z^{n}}{n}
$$

which is absolutely convergent for $|z|<1$ and is uniformly convergent for $|z|=1, z \neq 1$. Hence it has the boundary function denoted $l_{1}(z)$ with $z \neq 1$ on the unit circle: Writing $z=e^{2 \pi i x}, x \in \mathbb{R} \backslash \mathbb{Z}$, we have

$$
l_{1}(x)=\sum_{n=1}^{\infty} \frac{e^{2 \pi i n x}}{n}=-\log \left(1-e^{2 \pi i x}\right)
$$

often referred to as the Lerch zeta-function $l_{s}(x)$ at $s=1$. We shall make a full use of the identities

$$
\sum_{n=1}^{\infty} \frac{\cos (2 \pi n x)}{n}+i \sum_{n=1}^{\infty} \frac{\sin (2 \pi n x)}{n}=l_{1}(x)=\sum_{n=1}^{\infty} \frac{e^{2 \pi i n x}}{n}=A_{1}(x)-\pi i \bar{B}_{1}(x)
$$

$0<x<1$, where

$$
A_{1}(x)=-\log 2|\sin \pi x|=\sum_{n=1}^{\infty} \frac{\cos (2 \pi n x)}{n},
$$

is its real part, the Clausen function and the imaginary part is

$$
l_{1}(x)-l_{1}(-x)=-2 \pi i \bar{B}_{1}(x), 0<x<1,
$$

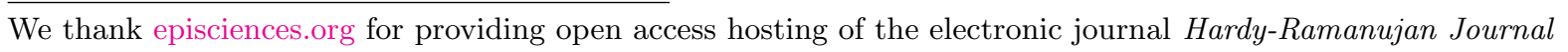


where

$$
\bar{B}_{1}(x)=x-[x]-\frac{1}{2}=\{x\}-\frac{1}{2}
$$

is the first periodic Bernoulli polynomial, and where $[x]$ indicates the greatest integer function and $\{x\}=x-[x]$ the fractional part of $x$. Since

$$
\bar{B}_{1}(x)=-\frac{1}{2} \sum_{n=1}^{\infty} \frac{\sin (2 \pi n x)}{n}
$$

for $x \notin \mathbb{Z}$, we often denote the saw-tooth Fourier series by $\psi(x)$, i.e.

$$
\psi(x)=\left\{\begin{array}{ll}
-\frac{1}{\pi} \sum_{m=1}^{\infty} \frac{\sin 2 \pi m x}{m}, & x \notin \mathbb{Z} \\
0, & x \in \mathbb{Z}
\end{array} .\right.
$$

Hence (1.8) and (1.6) differ at $x \in \mathbb{Z}$ by $\frac{1}{2}$, which affects Mordell's formula in his presentation (1.15).

It was Walfisz [Walf25], [Walf23] who first pointed out that the Fourier series (1.7) is a consequence of the functional equation for the Riemann zeta-function, the earliest occurrence of the modular relation, [Kats14].

It follows that

$$
l_{1}(x)=\sum_{n=1}^{\infty} \frac{e^{2 \pi i n x}}{n}=-\log 2|\sin \pi x|-\pi i \bar{B}_{1}(x) .
$$

We are naturally led to introduce the Lerch zeta-function which is the boundary function of the Hurwitz-Lerch transcendent:

$$
l_{s}(x)=\sum_{n=1}^{\infty} \frac{e^{2 \pi i n x}}{n^{s}}
$$

for $\sigma=\operatorname{Re} s>1, x \in \mathbb{R}$, say, cf. [LaGa02].

Our aim is to consider the Lambert-like series (with $b_{n}=\frac{1}{n}$ )

$$
f(x)=\sum_{n=1}^{\infty} b_{n} \frac{e^{2 \pi i n \beta}}{1-x e^{2 \pi i n \alpha}}
$$

and its associate

$$
F(x)=\sum_{n=-\infty}^{\infty} b_{n} \frac{e^{2 \pi i n \beta}}{1-x e^{2 \pi i n \alpha}}
$$

for $|x|<1$ in regard to the properties of the coefficients $\left\{b_{n}\right\}$ and the parameters $\alpha, \beta \in \mathbb{R}$. In the case $\sum_{n=-\infty}^{\infty}\left|b_{n}\right|<\infty, F(x)$ has an equivalent and more familiar expression

$$
F(x)=\sum_{n=0}^{\infty} g(\alpha n+\beta) x^{n}, \quad g(t)=\sum_{n=-\infty}^{\infty} b_{n} e^{2 \pi i n t} .
$$

A lot of investigations on the series (1.13) are concerned with the irrationality of the limit of the series, i.e. whether the limit is a rational function or not. Newman [New60], [Mor61], [Mor65a], [Mor65b], [New60] and [Hen67] are all devoted to the irraionality of the limit function and some are stated as Theorems $2.2 \sim 2.4$, [KuiNi74, pp. 10-13]. Other references can be found on [KuiNi74, p. $22]$ save for the paper of Henniger.

Mordell [Mor63] studied the convergence and the radial limit as $x \rightarrow 1$ of the imaginary part of the Lambert-like series (1.12) for $\alpha \notin \mathbb{Q},|x|<1$ in the case $\sum_{n=-\infty}^{\infty}\left|b_{n}\right|<\infty$. 
He introduced the auxiliary function (with $a_{n}=\frac{1}{n}$ )

$$
h(m, t)=\sum_{n=-\infty}^{\infty} a_{n} e^{2 \pi i m \alpha} t^{|n|}, \quad|t|<1,
$$

the prime on the summation sign indicating the omission of the term with $n=1$. For the special sequence $a_{n}=e^{2 \pi i n \beta}, b_{n}=\frac{e^{2 \pi i n \beta}}{n}$ Mordell [Mor63] established

$$
H(x)=\sum_{n=-\infty}^{\infty} \frac{e^{2 \pi i n \beta}}{n} \frac{1}{1-x e^{2 \pi i n \alpha}}=2 \pi i\left(\frac{1}{2(1-x)}-\sum_{m=0}^{\infty}\{\alpha m+\beta\} x^{m}\right)
$$

for $m \alpha+\beta \notin \mathbb{Z}$ and if $m \alpha+\beta$ is an integer, letting the value of $m$ be $k$, we must $-\pi x^{k}$ to the right-hand side of (1.15).

Davenport [Dav66] established a more general result in the case where $\sum_{n=-\infty}^{\infty} b_{n}$ is convergent.

Our main result is Theorem 1.1 below which gives the hidden whole entiity [Mor63] and implies that the left-hand side of (1.12) is the imaginary part of the Lambert-like series

$$
\tilde{g}(t, x)=\sum_{m=0}^{\infty} \tilde{h}(m, t) x^{m}=\sum_{n=1}^{\infty} a_{n}\left(\sum_{m=0}^{\infty} e^{2 \pi i m \alpha} x^{m}\right) t^{n},
$$

where correspondingly to $(1.14)$

$$
\tilde{h}(m, t)=\sum_{n=1}^{\infty} a_{n} e^{2 \pi i m \alpha} t^{n}
$$

for $|x|<1, \alpha \notin \mathbb{Q}$.

This is one of seeing the invisible project [CKK18] and is quite similar to the radial limit of a generalized Lambert series in the spirit of Riemann [Rie38b], cf. [Wan11, Theorem 3], [Wan18]. Cf. $\S 2$. for Lambert series.

Theorem 1.1. Let $\alpha \notin \mathbb{Q}, \beta$ real, $|x|<1$. Then

$$
\sum_{n=1}^{\infty} \frac{e^{2 \pi i \beta n}}{n}\left(\sum_{m=0}^{\infty} e^{2 \pi i m n \alpha} x^{m}\right)=\sum_{n=1}^{\infty} \frac{e^{2 \pi i n \beta}}{n} \frac{1}{1-x e^{2 \pi i n \alpha}}=\sum_{m=0}^{\infty} \tilde{h}(m, 1) x^{m},
$$

where

$$
\tilde{h}(m, 1)=-\log 2 \sin \theta(m)-\pi i \bar{B}_{1}(\theta(m))
$$

and where

$$
\theta(m)=\pi(m \alpha+\beta) \notin \mathbb{Z}
$$

By (1.9), (1.19) reads

$$
\tilde{h}(m, 1)=l_{1}(\theta(m)) .
$$

The key hinges on the threshold factor $\frac{1}{n}$ which is the gain obtained in Wintner's procedure of integration in $\S 2$..

In order to remove singularities, it is customary to use, as Riemann did, a well-known device of taking the odd part or an alternate sum described respectively by

$$
\sum_{2 \nmid n} a_{n}=\sum_{n} a_{n}-\sum_{2 \mid n} a_{n}
$$


or

$$
\sum_{n}(-1)^{n} a_{n}=\sum_{2 \mid n} a_{n}-\sum_{2 \nmid n} a_{n}=2 \sum_{2 \mid n} a_{n}-\sum_{n} a_{n},
$$

by (1.22), where $n$ runs over a finite range or the series are absolutely convergent.

As an example, incorporating the Bernoulli formula

$$
\psi(2 x)-\psi(x)=\psi\left(x+\frac{1}{2}\right)
$$

whose right-hand side is the Fourier series

$$
-\frac{1}{\pi} \sum_{n=1}^{\infty} \frac{1}{n} \sin 2 \pi n\left(x+\frac{1}{2}\right)
$$

we arrive at the trigonometric series of Riemann (cf. [Rie1854])

$$
\sum_{n=1}^{\infty} \frac{\psi\left(n x+\frac{1}{2}\right)}{n}=\sum_{n=1}^{\infty}\left(-\sum_{d \mid n}(-1)^{d}\right) \frac{\sin 2 \pi n x}{n \pi}
$$

for all point $x$.

\section{Lambert series}

In this section we extract some basics of Lambert series from

[LLWK17, pp. 131-136] and state the corrected version of the Dirichlet-Abel theorem, Theorem 2.1. Lambert series have been studied extensively since their introduction by Lambert [Lam1771]. Cf. [Win41], [KTY02], [Kats14, 73-80], [LLWK17, 131-136], [LiKa19] etc. Let $\left\{b_{n}\right\} \subset \mathbb{C}$ be such that

$$
\lim \sup \left|b_{n}\right|^{1 / n} \leq 1
$$

i.e. such that the power series $\sum_{n=1}^{\infty} b_{n} z^{n}$ is absolutely convergent in $|z|<1$. Then the Lambert series

$$
f(z)=\sum_{n=1}^{\infty} b_{n} \frac{z^{n}}{1-z^{n}}
$$

is absolutely convergent in $|z|<1$ and represents an analytic function and moreover the power series of this function can be obtained by formal rearrangement of (2.27), i.e.

$$
f(z)=\sum_{n=1}^{\infty} a_{n} z^{n} \quad(|z|<1)
$$

the Liouville formula, where

$$
a_{n}=\sum_{d \mid n} b_{d}
$$

Along with (2.27), one may also consider a lá Wintner [Win41]

$$
f_{+}(z):=\sum_{n=1}^{\infty} \beta_{n} \frac{z^{n}}{1+z^{n}}
$$

One of the most prominent occurrence of Lambert series is the celebrated formula of Ramanujan which provides an expression of zeta-values in terms of rapidly converging Lamber series, which is elucidated as a modular relation [KTY02], [Kats14]. 
Another instance is eleuciadation of Riemann's posthumous Fragment II all the results of which deal with the asymptotic behavior of those modular functions from Jacobi's Fundamenta Nova [Jac], for which the variable tends to rational points on the unit circle. Those formulas were simply noted for record by Riemann. Dedekind [Ded1892] was the first who analyzed Fragment II and introduced the most famous Dedekind eta-function (and the Dedekind sum). Several authors including Smith [Smi1881], Hardy [Har03], Rademacher [Rad31] Bayad [Bay01] made some more incorporations of Fragment II. More recently in 2004, Arias-de-Reyna [dR04] spent 67 pages to analyze all the formulas in Fragment II by ad hoc method.

Wang [Wan11], [Wan18] chose a suitable $R$-function in taking the radial limits and applied a form of Dirichlet-Abel Theorem, Theorem 2.1, whereby he almost immediately got the expression in terms of the (differences of) polylogarithm function of order 1, condensing 67 to 17 pages. This has been further simplified [WAK20]. This is precisely what Riemann intended to do, i.e. to eliminate the singular part, which turns out to be the Clausen function, by taking the odd part. Riemann must have felt the truth of this type of Dirichlet-Abel theorem. The following is the corrected version of the theorem in [Wan11]:

Theorem 2.1. (Dirichlet-Abel) Let $q$ be a fixed modulus $>1$. Let $R_{n}(x)$ denote a complex-valued function defined on $\mathrm{I}=[0,1]$ such that $R_{n}(x)=R_{k}(x)$ for $n \equiv k(\bmod q)$, and a fortiori, there are $q$ different functions. Assume that each $R_{k}(x)$ is of Lipschitz $\alpha, \alpha \geq 1$, [in symbol $R_{k}(x) \in \operatorname{Lip} \alpha$ ] and the vanishingness condition

$$
\sum_{k=1}^{q} R_{k}(x)=0
$$

for each $x \in \mathrm{I}$. Then the Dirichlet series

$$
F(s, x)=\sum_{n=1}^{\infty} \frac{R_{n}\left(x^{n}\right)}{n^{s}}
$$

is uniformly convergent in $\operatorname{Re} s=\sigma>0$ and $x \in \mathrm{I}$.

If, further, $R_{k}$ are all continuous on $\mathrm{I}$, then $F(s)$ is also continuous on $\mathrm{I}$ and

$$
F(1,1)=\sum_{n=1}^{\infty} \frac{R_{n}(1)}{n}=-\frac{1}{q} \sum_{k=1}^{q} R_{k}(1) \psi\left(\frac{k}{q}\right)=\sum_{k=1}^{q} \hat{R}_{k}(1) l_{1}\left(\frac{k}{q}\right),
$$

where $l_{1}(x)$ is (1.2) and $\psi$ is the Euler digamma function

$$
\psi(x)=\frac{\Gamma^{\prime}}{\Gamma}(x)
$$

For a Dirichlet series $G(s)=\sum_{n=1}^{\infty} \frac{a_{n}}{n^{s}}$ with periodic coefficients $\left\{a_{n}\right\}$ of period $q,(2.33)$ reads

$$
\lim _{s \rightarrow 1}\left(G(s)-\frac{\frac{\hat{a}_{q}}{\sqrt{q}}}{s-1}\right)=-\frac{1}{q} \sum_{k=1}^{q} a_{k} \psi\left(\frac{k}{q}\right)=\sum_{k=1}^{q-1} \hat{a}_{k} \ell_{1}\left(\frac{k}{q}\right)+\frac{\hat{a}_{q}}{\sqrt{q}} \gamma
$$

where

$$
\hat{a}_{n}=\frac{1}{\sqrt{q}} \sum_{k=1}^{q} e^{-2 \pi i k \frac{n}{q}} a_{k}, \quad \hat{a}_{q}=\frac{1}{\sqrt{q}} \sum_{k=1}^{q} a_{k} .
$$


For the moment we shall dwell on Wintner's elucidating of Riemann's procedure in Fragment I, [Win41]. Riemann divides the Lambert series (2.28) by $z$ and integrate the result from 0 to $z=r e^{i \theta}$, $r=|z|<1$, and then puts $r=1$ to obtain his results, i.e. "Fourier expansions", in a formal way. Wintner's legitimation reads as follows. Slightly modifying Riemann's procedure by the trivial factor $e^{i \theta}$, which makes the function $F=F(r, \theta)$ a function in $z$, one obtains

$$
F(z)=\int_{0}^{r} \frac{f\left(r e^{i \theta}\right)}{r} \mathrm{~d} r=\sum_{n=0}^{\infty} c_{n} z^{n},
$$

with

$$
c_{n}=\frac{1}{n} b_{n}=\frac{1}{n} \sum_{d \mid n} a_{n} .
$$

In the case of $(2.27)$, we should have

$$
c_{n}=\frac{1}{n} \sum_{d \mid n}(-1)^{n} a_{n}
$$

Then Wintner asks if $F(z)$ tends to a measurable boundary function $F\left(e^{i \theta}\right)$ as $r \rightarrow 1$ (within the Stolz path) and if so, then whether or not the boundary function is of class $L^{p}$, so that Riemann's formal trigonometric series actually is the Fourier series of the boundary function. This approacb to bundary functions is to be compared with the inner function concept in [Beu49]. They form basis of study of boundary behavior [LLK19].

The $L^{p}$-condition

$$
\sum_{n=-\infty}^{\infty}\left|c_{n}\right|^{p /(p-1)}<\infty \quad(p \geq 2)
$$

may be used for an $f$ to be of class $L^{p}$ for some $p \geq 2$.

Theorem 2.2. (Wintner) Suppose

$$
a_{n}=O\left(n^{\lambda-\delta}\right)
$$

for some $\delta>0$ and a fixed $0<\lambda \leq \frac{1}{2}$. Then the boundary function $F\left(e^{i \theta}\right)$ exists and is measurable such that

$$
F\left(r e^{i \theta}\right) \rightarrow F\left(e^{i \theta}\right) \quad \text { a.e., as } r \rightarrow 1
$$

along the Stoltz path. If $\lambda<\frac{1}{2}$, then $F\left(e^{i \theta}\right)$ is of class $L^{1 / \lambda}$ and if in (2.41), the exponent can be taken arbitrarily small, then it is of class $L^{\infty}$.

Example 2.3. The case $a_{n}=1$ is the Lambert series considered by Lambert himself ([Lam1771]), (2.28) resp. (2.37) reads

$$
f(z)=\sum_{n=1}^{\infty} \frac{z^{n}}{1-z^{n}}=\sum_{n=1}^{\infty} d(n) z^{n}, \quad|z|<1,
$$

resp.

$$
F(z)=\sum_{n=1}^{\infty} \frac{d(n)}{n} z^{n}, \quad|z|<1,
$$

where $d(n)$ indicates the number of all positive divisors of $n$. It is well-known that

$$
d(n)=O\left(n^{\varepsilon}\right), \quad \forall \varepsilon>0 .
$$

Hence by Theorem 2.2, the boundary function $F\left(e^{i \theta}\right)$ exists and

$$
F\left(e^{i \theta}\right)=\sum_{n=0}^{\infty} \frac{d(n)}{n} e^{i n \theta} \quad \text { a.e. }
$$


Remark 2.4. We note that the imaginary part of (2.46) gives the expansion treated in [Win37], which in turn gives rise to the series considered by Riemann [Rie38b]. Indeed, this was proved by Chowla and Walfisz [ChWa34]. Cf. [Kats14, p. 198].

Furthermore, by employing a method in [LMZ10] we may prove the trigonometric identity

$$
\sum_{n=1}^{\infty} \frac{1}{n^{z}} \bar{B}_{l}\left(n x+\frac{1}{2}\right)=-\frac{l !}{2 \pi i} \sum_{\substack{n=-\infty \\ n \neq 0}}^{\infty} \frac{\sigma_{l-z}^{*}(n)}{n^{l}} e^{2 \pi i n x}
$$

where $\bar{B}_{l}(x)$ is the $l$-th periodic Bernoulli polynomial whose special case reduces to Riemann's formula (1.25).

\section{Elucidation of Mordell's identity}

We shall reveal that Mordell's formula may be treated exactly as in the case of the limit values of elliptic modular functions in Riemann's fragment II evaluated by the differences of monologarithm function $l_{1}(x)$ whose right-hand side is the Fourier series

$$
-\frac{1}{\pi} \sum_{n=1}^{\infty} \frac{1}{n} \sin 2 \pi n\left(x+\frac{1}{2}\right)
$$

which is $\bar{B}_{1}\left(x+\frac{1}{2}\right)$ for $x \notin \mathbb{Z}$ and is 0 for $x \in \mathbb{Z}+\frac{1}{2}$.

Theorem 3.1. (Tauberian theorem) Suppose $\tilde{g}(t, x)$ is convergent uniformly in $t, 0 \leq t \leq 1$. Then if $a_{n}=O\left(\frac{1}{n}\right)$, then for $|x|<1$ the series

$$
\sum_{m=0}^{\infty} \sum_{n=1}^{\infty} a_{n} e^{2 \pi i m n \alpha} x^{m}=\sum_{n=1}^{\infty} a_{n} \frac{1}{1-x e^{2 \pi i n \alpha}}
$$

converges to $\tilde{g}(1, x)=\sum_{m=0}^{\infty} \tilde{h}(m, 1) x^{m}$.

This follows from Littlewood's Tauberian theory [Har, Theorem 90, p. 154], [Kor04, p. 14] as in [Mor63].

In view of (1.5), we may consider the imaginary part of $\tilde{h}(m, t)$, which is the function $h(m, t)=$ $2 i \operatorname{Im} \tilde{h}(m, t)$, where $h(m, t)$ resp. $\tilde{h}(m, t)$ are defined by (1.14) resp. (1.16).

This proves Theorem 1.1 and Mordell's equality [Mor63, (14)] is to read

$$
\begin{aligned}
H(x) & =\sum_{n=-\infty}^{\infty} \frac{e^{2 \pi i n \beta}}{n} \frac{1}{1-x e^{2 \pi i n \alpha}}=\sum_{n=-\infty}^{\infty} \frac{e^{2 \pi i \beta n}}{n}\left(\sum_{m=0}^{\infty} e^{2 \pi i m n \alpha} x^{m}\right) \\
& =2 \pi i \sum_{m=0}^{\infty} \bar{B}_{1}(m \alpha+\beta) x^{m} .
\end{aligned}
$$

Indeed, if $\alpha m+\beta \in \mathbb{Z}$, then $\{\alpha m+\beta\}=0$ and since on the right-hand side the corresponding term is to be $-\pi\left(\{\alpha m+\beta\}-\frac{1}{2}\right) x^{k}=\frac{\pi}{2} x^{k}$, we must add this. This clumsiness is removed by adopting the periodic first Bernoulli polynomial as in (3.48).

However, to deduce (1.15), we are to appeal to (1.6). The asymptotic behavior of $H(x)$ can be obtained from the following lemma with $g(m)=\bar{B}(\alpha m+\beta)$. 
Lemma 3.2. Let

$$
M(g)=\lim _{N \rightarrow \infty} \frac{1}{N+1} \sum_{m=0}^{N} g(m),
$$

which is equivalent to

$$
G(N):=\sum_{m=0}^{N} g(m)=M(g)(N+1)+o(N) .
$$

Then for $|x|<1$, we have as $x \rightarrow 1-0$,

$$
\sum_{m=0}^{\infty} g(m) x^{m}=M(g) \frac{1}{1-x}+o\left(\frac{1}{1-x}\right) .
$$

Proof. By partial summation

$$
\begin{aligned}
& \sum_{m=0}^{\infty} g(m) x^{m}=\lim _{N \rightarrow \infty}\left(G(0)+\sum_{m=1}^{N}(G(m)-G(m-1)) x^{m}\right) \\
& =\lim _{N \rightarrow \infty}\left(\sum_{m=0}^{N-1} G(m)\left(x^{m}-x^{m+1}\right)+G(N) x^{N}\right) \\
& =(1-x) M(g) \sum_{m=0}^{\infty}(m+1) x^{m}+o\left((1-x) \sum_{m=0}^{\infty} m|x|^{m}\right)
\end{aligned}
$$

on substituting from (3.49). Rcalling the differentiated geometric series

$$
\sum_{m=1}^{\infty} m x^{m-1}=\frac{1}{(1-x)^{2}}
$$

whence (3.50) follows.

Mordell deduces that as $x e^{2 \pi i l \beta} \rightarrow 1$,

$$
H(x)=\frac{e^{2 \pi i l \beta}}{l} \frac{1}{1-x e^{2 \pi i l \alpha}}+o\left(\frac{1}{\left|1-x e^{2 \pi i l \alpha}\right|}\right)
$$

This gives a solution to [KuiNi74, Exercise 2.20] that $H(x)$ has the unit circel as its natural boundary when $\alpha \notin \mathbb{Q}$.

As a radial limit in $\S 2$. we are to consider

$$
H_{r}(x)=\sum_{n=-\infty}^{\infty} \frac{r^{n} e^{2 \pi i n \beta}}{n} \frac{1}{1-x e^{2 \pi i n \alpha}}
$$

as $r \rightarrow 1-0$.

Concluding remarks: Lehmer [Leh75] made use of both real and imaginary parts of the monologuefunction (1.3) in connection with his study on generalized Euler constants, cf. [WAK20]. There we state a general principle to the effect that Laurent coefficients is that which governs the situation including Kronecker limit type formulas. 
In contrast to these, Titchmarsh [Tit38, p. 253] treats the case of the Clausen function:

$$
F_{\nu}(z)=\sum_{n=1}^{\infty} d_{\nu}(n) e^{2 \pi i n z}
$$

as $z$ approaches a rational point of the unit circle. This can be studied in the context of the product of Hurwitz and Lerch zeta-functions considered by Estermann [Est29a], [Est29b], [Est30] as has been done by Chowla and Walfisz [ChWa34]. More general study on these zeta-functions that satisfy the ramified functional equaitions will be conducted elsewhere.

The Liouville formula $(2.30)$ is

$$
f_{+}(z)=\sum_{m=1}^{\infty} \sum_{n=1}^{\infty}(-1)^{m-1} \beta_{n}\left(z^{m}\right)^{n}=\sum_{\ell=1}^{\infty} \alpha_{\ell} z^{\ell},
$$

where

$$
\alpha_{n}=-\sum_{d \mid n}(-1)^{d} \beta_{n / d} .
$$

Hence the generating Dirichlet series for $\mathrm{A}=\left\{\alpha_{n}\right\}$

$$
\varphi_{+}(s)=\varphi_{\mathrm{A},+}(s)=\sum_{n=1}^{\infty} \frac{\alpha_{n}}{n^{s}}
$$

has the expression

$$
\varphi_{+}(s)=l_{s}\left(\frac{1}{2}\right) \varphi_{\mathrm{B}}(s)
$$

where

$$
\varphi_{B}(s)=\sum_{n=1}^{\infty} \frac{b_{n}}{n^{s}} .
$$

We shall conduct the study of these zeta-functions elsewhere.

Acknowledgment. The authors would like to thank the referee for careful reading of the earlier draft.

\section{References}

[Bay01] A. Bayad, Sommes de Dedekind elliptiques et formes de Jacobi, Ann. Inst. Fourier, Tome 51 (2001, $29-42$.

[Beu49] A. Beurling, On two problems concerning linear transformations in Hilbert space, Acta Math. 81 (1949), 239-255.

[Cam66] R. Campbell, Les intégrales Eulérienne et leurs applications, étude apprpoondie de la fonction gamma, Dunod, Paris, 1966.

[CKK18] K. Chakraborty, S. Kanemitsu, and T. Kuzumaki, Seeing the invisible: around generalized Kubert functions, Annales Univ. Sci. Budapest., Sect. Comp. 47 (2018), 185-195.

[CKT09] K. Chakraborty, S. Kanemitsu and H. Tsukada, Vistas of Special Functions II, World Scientific, London-SingaporeNew Jersey, 2009.

[ChWa34] S. Chowla and A. Walfisz, On a trigonometric sum, Proc. London Math. Soc. 34 (1934), 401-413; The Collected Papers of Sarvadaman Chowla I, CRM, (1999), 267-279.

[Dav66] H. Davenport, Note on an irrational power series, Proc. Amer. Math. Soc. 17 (1966), 1-5; Collected works of Harold Davenport IV, 1806-1810, Academic Press 1977.

[Ded1892] R. Dedekind, Erläuterungen zu zwei Fragmenten von Riemann, Math. Werke Bd. 1, 159-173, Braunschweich, (1930) (In: Bernhard Riemanns gesammelte mathematische Werke und wissenschaftlichen Nachlass, 2, Aufl., 466-478, (1892)). 
[dR04] A. de Reyna, Riemann's Fragment on the limit values of elliptic modular functions. Ramanujan J. 8 (2004), $57-123$.

[Est29a] T. Estermann, Vereinfacher Beweis eines Satzes von Kloosterman, Hamburger Abh. 7 (1929), 83-98.

[Est29b] T. Estermann, On the representation of a number as the sum of three products, Proc. London Math. Soc. 29 (1929), $453-478$.

[Est30] T. Estermann, On the representation of a number as the sum of two products, Proc. London Math. Soc. 31 (1930), $123-133$.

[Har03] G. H. Hardy, Note on the limiting values of the elliptic modular-functions, Quart. J. Math. 34 (1903), 76-86; Collected Papers of G. H. Hardy IV, OUP, Oxford 1966, 251-261.

[Har] G.H.Hardy, Divergent series, OUP, Oxford,1956.

[Hen67] J. Henniger, Power series with almost periodic coefficients, Proc. Amer. Math. Soc. 18 (1967), 53-56.

[IsKa99] M. Ishibashi and S. Kanemitsu, Dirichlet series with periodic coefficients, Res. Math. 35 (1999), $70-88$.

[Jac] C. G. J. Jacobi, Fundamenta nova, §40 (Ges. Werke Vol. 1, 159-164).

[Jut87] M. Jutila, Lectures on a method in the theory of exponential sums, TIFR Mumbai, 1987.

[Kan87] S. Kanemitsu, Evaluation of certain limits in closed form, Proc. Intern. Conf. Number Theory, Univ. Laval, Ed. by de Koninck and Levesque, de Gruyter, 1987, 459-474.

[KTY02] S. Kanemitsu, Y. Tanigawa, and M. Yoshimoto, Ramanujan's formula and modular forms, Number-theoretic Methods - Future Trends, Proceedings of a conference held in Iizuka, Ed. by S. Kanemitsu and C. Jia, Kluwer, Dordrecht, 2002, 159-212.

[KaTs07] S. Kanemitsu, and H. Tsukada, Vistas of Special Functions, World Scientific, Singapore, 2007.

[Kats14] S. Kanemitsu and H. Tsukada, Contributions to the theory of zeta-functions: the modular relation supremacy, World Scientific, Singapore, 2014.

[Kor04] J. Korevaar, Tauberian theory, A century of developments, Springer, Berlin-Heidelberg-New York, 2004.

[KuiNi74] L. Kuipers and H. Niederreiter, Uniform distribution of sequences, Wiley, New York, 1974.

[Lam1771] J.H. Lambert, Anlage zur Archtektonik oder Theorie des Einfachen und Ersten in der philosophischen und mathematischen Erkenntnis, 2 Bände, Riga, Vol. 2, §875 (p.507), 1771.

[LaGa02] A. Laurinchikas and R. Garunkstis, The Lerch zeta-Function, Kluwer Academic Publ., Dordrecht-Boston-London 2002.

[Leh75] D. H. Lehmer, Euler constants for arithmetic progressions, Acta Arith. 27 (1975), 125-142; Selected Papers of D. H. Lehmer, Vol. II, 591-608, Charles Babbage Res. Center, Manitoba 1981.

[LiKa19] F.H.Li and S. Kanemitsu, Around boundary functions of the right half-plane and the unit disc, Advances in Special Functions and Analysis of Differential Equations, CRC Press, Taylor \& Francis, 2020, (to appear).

[LLK19] H. Y. Li, F. H. Li and S. Kanemitsu, The ideas and use of meta-science, Proc. Intern. Conf. "Recent Developments in Engineering \& Technology (ICRDET-2019)" at Anand International College of Engineering, Jaipur, Sep. 14-15, 2019, (to appear).

[LLWK17] H. L. Li, F.H. Li, N.L. Wang and S. Kanemitsu, Number theory and its applications II, 285 pages, World Scientific, London-Singapore-New Jersey, 2017.

[LMZ10] H.L. Li, J. Ma and W.P.Zhang, On some Diophantine Fourier series, Acta Math. Sinica, English Ser. 26 (2010), 1125-1132

[Mil83] J. Milnor, On polylogarithms, Hurwitz zeta-functions and the Kubert identities, Enseign. Math. (2) 29 (1983), 281-322.

[Mor61] L. J. Mordell, Irrational power series I, Proc. Amer. Math. Soc. 12 (1961), 522-526.

[Mor63] L. J. Mordell, The series $\sum a_{n} /\left(1-e^{2 \pi i n \alpha}\right)$, J. London Math. Soc. 38 (1963), 111-116.

[Mor65a] L. J. Mordell, Irrational power series II, Acta Arith. 11 (1965), 181-188.

[Mor65b] L. J. Mordell, Irrational power series III, Proc. Amer. Math. Soc. 16 (1965), 819-821.

[New60] M. Newman, Irrational power series, Proc. Amer. Math. Soc. 12 (1960), 699-702.

[Rad31] H. Rademacher, Zur Theorie der Modulfunktionen, J. Reine Angew. Math. 167 (1931, )312-336; Collected Papers of H. Rademacher I, 652-677.

[Rad73] H. Rademacher, Collected Papers of Hans Rademacher I, II, MIT Press, Cambridge, Mass,1973.

[Rie38a] B. Riemann, Collected Works of Bernhard Riemann, ed. by H. Weber, 2nd ed. Dover, New York,1938.

[Rie38b] B. Riemann, Fragmente über Grenzfälle der ellipitischen Modulfunctionen, in [Rie38a], 455-465, 1938.

[Rie1854] B. Riemann, Über die Darstellbarkeit einer Function durch eine trigonometrische Reihe, in Riemann's Collected works (1854), pp 227-265.

[Smi1881] H.S. J.Smith, On some discontinuous series considered by Riemann, Mess. Math. (2) 11 (1881), 1-11; Collected Papers Vol. 2 (1966), 312-320. 
[SrCh01] H. M. Srivastava and J.S. Choi, Series associated with the Zeta and related functions, Kluwer Academic Publishers, Dordrecht-Boston-London, 2001.

[Tit38] E. C. Titchmarsh, On a series of Lambert's type, J. London Math. Soc. 13 (1938), 248-252.

[Walf23] A.Z. Walfisz, Über die summatorischen Funktionen einiger Dirichletscher Reihen, Inaugural Diss. Göttingnen, 1923.

[Walf25] A. Z.Walfisz, Über das Piltzsche Teilerproblem in algebraishcen Zahlkörpern, Math. Z. 22 (1925), 153-188.

[Wan11] N. L. Wang, On Riemann's posthumous Fragment II on the limit values of elliptic modular functions, Ramanujan J., 24 (2011), 129-145.

[Wan18] N. L.Wang, Arithmetical Fourier and limit values of elliptic modular functions, Proc. Math. Sci. 128: 28, 2018. https://doi.org/10.1007/s12044-018-0408-1.

[WAK20] N.L.Wang, P. Agarwal and S. Kanemitsu, Limiting values and functional and difference equations, Special issue on Nonlinear Equations: Theory, Methods, and Applications, Mathematics 2020, 8, 407; doi:10.3390/math8030407 24 pages.

[WMK20] X.H.Wang, J.Mehta, and S.Kanemitsu, The boundary Lerch zeta-function and short character sums a lá Y. Yamamoto, Kyushu J. Math. (2020), (to appear).

[Win37] A. Wintner, On a trigonometrical series of Riemann, Amer. J. Math. 59 (1937), 629-634.

[Win41] A. Wintner, On Riemann's fragment concerning elliptic modular functions, Amer. J. Math. 63 (1941), $628-634$.

\section{P. Agarwal}

Anand International College Engineering

Jaipur, India

e-mail: goyal.praveen2011@gmail.com

\section{S. Kanemitsu}

Graduate School of Engineering

Kyushu Institute of Technology

Kitakyushu 804-8555, Japan

e-mail: omnikanemitsu@yahoo.com

\section{T. Kuzumaki}

Faculty of Engineering

Gifu University

Gifu 501-1193, Japan

e-mail: kuzumaki@gifu-u.ac.jp 